\title{
Letter from the Editor - in - Chief
}

In this edition we publish five papers. The first three papers have been chosen by the program committee of 2005 Brazilian Symposium on Computer Graphics \& Image Processing (SIBGRAPI05) and 2004 Brazilian Symposium on Formal Methods (SBMF04) to be published in the JBCS in an extended and reviewed format.

The first paper by Thomaz, Kitani and Gillies presents a maximum uncertainty Linear Discriminant Analysis (LDA) based approach for limited sample size problems with application to face recognition. It is based on a straightforward stabilisation approach for the withinclass scatter matrix. The classification results indicate that the new method improves the LDA classification performance when the within-class scatter matrix is not only singular but also poorly estimated, with or without a Principal Component Analysis intermediate step and using less linear discriminant features.

The second paper by Fernandes, Oliveira, Silva and Crespo presents a new method for computing dimensions of boxes from single-perspective projection images in real time. The approach is based on projective geometry and computes the box dimensions using data extracted from the box silhouette and from the projection of two parallel laser beams on one of the imaged faces of the box. Also, the paper presents the construction of a scanner prototype, a complete discussion about the performed measurements, and an error propagation analysis that allows the method to estimate the uncertainty associated to all measurements made over each frame of a video sequence, in real-time.

The third paper by Khoumsi addresses the issue of test synthesis (or test generation) which can be described as follows: from a formal specification of an implementation under test (IUT), and from a test purpose describing behaviors to be tested, the aim is to synthesize test cases to be executed in order to check whether the IUT conforms to its formal specification, while trying to control the IUT so that it satisfies the test purpose. The work studies the synthesis of test cases for symbolic real-time systems. The method presented combines and generalizes two testing methods presented in previous work, namely: 1) a method for synthesizing test cases for (non-symbolic) real-time systems, and 2) a method for synthesizing test cases for (non-real-time) symbolic systems.

The last two papers have been submitted to the JBCS. The fourth paper by Vincenzi, Simão, Delamaro and Maldonado presents a testing process based on mutation testing, named Muta-Pro. Mutation testing criterion is considered very effective in detecting faults but it has a high cost of application due to i) the large number of generated mutants; ii) the time-consuming activity of determining equivalent mutants; and iii) the mutant execution time. Muta-Pro integrates different researches aiming at allowing the application of mutation testing with a lower cost, keeping its effectiveness on detecting faults. The main purpose is to integrate MutaPro in an automated testing environment to promote its practical use.

The fifth paper by Rosa and Cunha adopts software architecture principles for structuring middleware specifications together with LOTOS for formalising their behaviour. The adoption of software architecture principles makes explicit structural aspects of the middleware, while the formalisttion enables us to check behavioural properties of the middleware.

I would like to express my gratitude to chairs of SIBGRAPIO5 and SBMFO4 as well as their program committee members for devoting their time to select the best papers among the many presented in the events. Last but not least, our thanks to the many anonymous reviewers that dedicated their time to the Journal of the Brazilian Computer Society.

Jaelson F. B. Castro 